\title{
Bloqueo plano erector de la espina en toracotomía pediátrica
}

\section{Pediatric thoracotomy in erector spinae plane block}

\author{
Luis Eduardo Misas Cobilla', Amelia Meza Acevedo2, ${ }^{*}$, Orlando Carlos Ochoa García ${ }^{3}$, Camilo Florez Acevedo, \\ Víctor Humberto Guerrero ${ }^{5}$ \\ Médico Residente III año Posgrado de Anestesiología y Reanimación Universidad de Cartagena. \\ Médico interno, Universidad de Cartagena. \\ Anestesiólogo, Fundación Hospital Infantil Napoleón. \\ Médico Epidemiólogo. Universidad CES. \\ 5 Anestesiólogo Cardiovascular. Universidad CES.
}

Fecha de recepción:30 de agosto de 2020 / Fecha de aceptación:15 de septiembre de 2020

\begin{abstract}
Erector spinae plane block (ESP) is a regional anesthesia technique consisting of blocking the interfascial plane, where local anesthetic (LA) is injected at the site where the dorsal branch of the spinal nerve emerges. There are various publications on the application of the block (EPS) in the adult population, however there are few reports of the use of this block in pediatric surgery. We present the first case report of an infant under 9 months of age with the presence of a tumor in the anterior mediastinum, who underwent a resection through the 6th intercostal space, previous blockage of the erector spinae under ultrasound vision. The patient did not present adverse effects and was discharged on the fourth postoperative day.
\end{abstract}

Key words: Anesthesia, conduction, pediatrics, mediastinal neoplasms, thoracotomy.

\section{RESUMEN}

El bloqueo del plano erector de la espina (ESP) es una técnica de anestesia regional consistente en bloquear el plano interfascial, donde se inyecta anestésico local (LA) en el sitio donde emerge la rama dorsal del nervio espinal. Existen diversas publicaciones sobre la aplicación del bloqueo (EPS) en población adulta, sin embargo, hay pocos reportes del uso de este bloqueo en cirugía pediátrica. Presentamos el primer reporte de caso de una lactante menor de 9 meses de edad con presencia de tumor en mediastino anterior, al cual se le hizo una resección a través del $6^{\circ}$ espacio intercostal, previo bloqueo del erector de la espina bajo visión ecográfica. La paciente no presentó efectos adversos y fue dada de alta al cuarto día posoperatorio.

Palabras clave: Anestesia regional, pediatría, neoplasias del mediastino, toracotomía.

amezaa2@unicartagena.edu.co

*ORCID: https://orcid.org/ 


\section{Introducción}

E: I bloqueo del plano erector de la espina (ESP) es una técnica de anestesia regional reciente descrita por primera vez en septiembre de 2016 por Forero et al. en el dolor neuropático severo a nivel de T5[1]. Esta técnica consiste en el bloqueo del plano interfascial, donde se inyecta anestésico local (LA) en el sitio donde emerge la rama dorsal del nervio espinal, es decir, entre el músculo erector de la columna espinal y el proceso trasverso, proporcionando anestesia al tórax en su región posterior anterior y lateral[1]-[3].

Su efecto anestésico es dado básicamente por la difusión del LA, a través de los tejidos blandos intertransverso al espacio paravertebral, alcanzando las ramas ventrales, dorsales y comunicantes de los nervios espinales[1]-[3].

El bloqueo ESP se ha hecho popular entre los anestesiólogos, en primera instancia porque el músculo erector de la columna vertebral se extiende a nivel toracolumbar[1], permitiendo una extensión céfalo-caudal y la cobertura de múltiples dermatomas[1],[3],[4], adicionalmente, es una técnica sencilla y relativamente segura, que permite fácilmente la identificación anatómica con ultrasonido[1],[3], este último a la vez sirve como guía para administrar el volumen necesario de LA, evitando la toxicidad; finalmente, no existen riesgos de lesión de estructuras vecinas[1] porque el lugar donde se introduce la aguja está alejado de la pleura y las estructuras vasculares principales[3], disminuyendo el riesgo de daño neurológico y neumotórax[2].

Actualmente, han surgido publicaciones sobre la aplicación del bloqueo del plano del músculo erector de la columna espinal en población adulta, no obstante, existen pocos reportes del uso de este bloqueo en cirugía pediátrica. A continuación, se presenta un reporte de caso de una lactante menor de 9 meses de edad con presencia de tumor en mediastino anterior.

\section{Caso clínico}

Lactante menor de 9 meses de edad, nacida a término, siendo producto de segundo embarazo por parto vaginal sin antecedentes relevantes. Ingresa programada para resección de tumor en mediastino anterior por toracotomía anterolateral izquierda (a nivel de 6 espacio intercostal). Duración de procedimiento de $3 \mathrm{~h}$, resección de tumor de $6 \mathrm{~cm}$ de diámetro. Equipo quirúrgico no solicita ventilación monopulmonar por lo que se intuba sin incidentes con tubo estándar, sin bloqueador bronquial. Los signos vitales al ingreso se muestran en la Figura 1 .

Inducción anestésica con propofol $3 \mathrm{mg} / \mathrm{kg}$, remifentanilo 2 $\mathrm{mg} / \mathrm{kg}$ y dexametasona $2 \mathrm{mg}$, mantenimiento con sevofluorano a 0,6 mac e infusión de remifentanilo 0,2 $\mathrm{mcg} / \mathrm{kg} / \mathrm{min}$, analgesia multimodal con dipirona $350 \mathrm{mg}$ y tramadol $10 \mathrm{mg}$.

Posicionada en decúbito lateral derecho presentó sangrado intraoperatorio de aproximadamente 250 cc por lo que se transfundió con 1 unidad de glóbulos rojos. Al finalizar la cirugía se colocó tubo de toracostomía $N^{\circ} 12$ a través del $6^{\circ}$ espacio intercostal izquierdo más trampa de agua a $-5 \mathrm{~cm} \mathrm{H}_{2} \mathrm{O}$.

Previamente y bajo anestesia general se realizó marcaje de zona de bloqueo para facilitar procedimiento (Figura 2).

Previa asepsia y antisepsia se identificó imagen objetivo con planos anatómicos clave (tejido celular subcutáneo, músculo

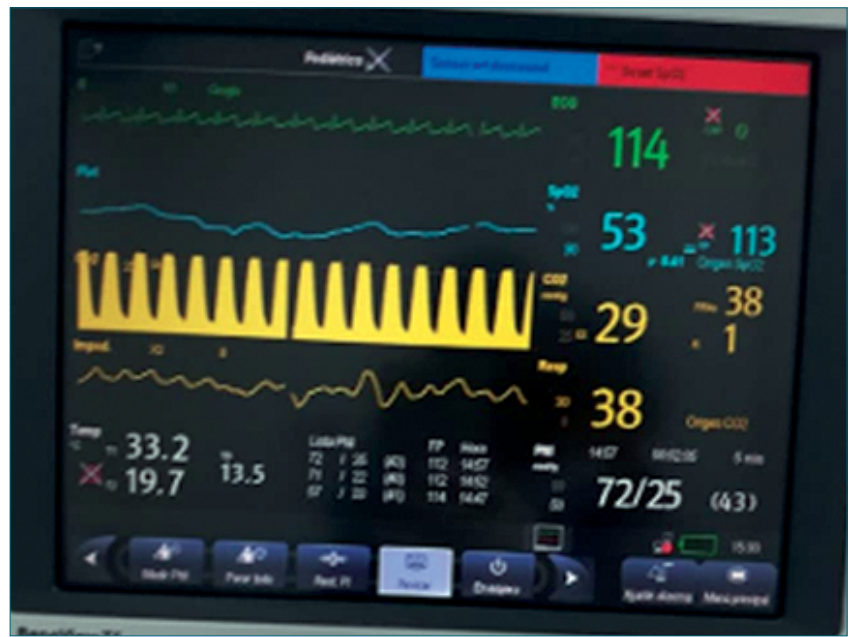

Figura 1. Signos vitales de ingreso (peso: $7 \mathrm{~kg}$ ) (fuente: los autores).

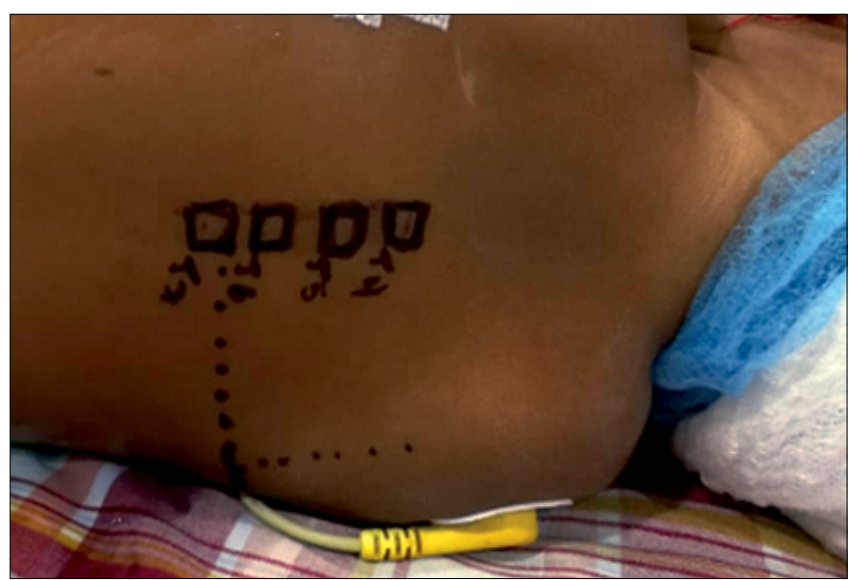

Figura 2. Plano superficial intercostal y ubicación de T6 (fuente: los autores).

trapecio, romboide mayor, erector de la espina y proceso transverso de T6), se realizó bloqueo erector de la espina bajo visión ecográfica con transductor lineal de $10 \mathrm{MHz}$ (Figura 3). Se identificó proceso transverso de T6 con transductor en posición sagital paramedial izquierda y se puncionó en plano con aguja Stimuplex ${ }^{\circledR}$ D 50 mm en dirección céfalo-caudal, contactando con proceso transverso. Tras aspiración negativa se administraron 7,5 ml de bupivacaína con epinefrina al 0,25\%, sin encontrar cambios en los parámetros de monitoría no invasiva.

Posterior a ello, la paciente fue extubada y transferida a la UCIP en buenas condiciones generales, tranquila y sin llanto, escala FLACC 0/10 (Figura 4).

Se realizó primer control de dolor en las $3 \mathrm{~h}$ posteriores a ingreso a UCIP, paciente con FLACC 0/10.

Nuevo control a las $12 \mathrm{~h}$ paciente con FLACC 0/10, estable hemodinámicamente, sin soporte ventilatorio, analgesia endovenosa suplementaria sin opioides, dipirona 350 mg cada 8 horas (IV).

Se reinició vía oral a las 4 horas de posquirúrgico sin problemas. A las $24 \mathrm{~h}$ se mantuvo estable, sin requerimientos de oxígeno, con dolor controlado mediante analgesia endovenosa 


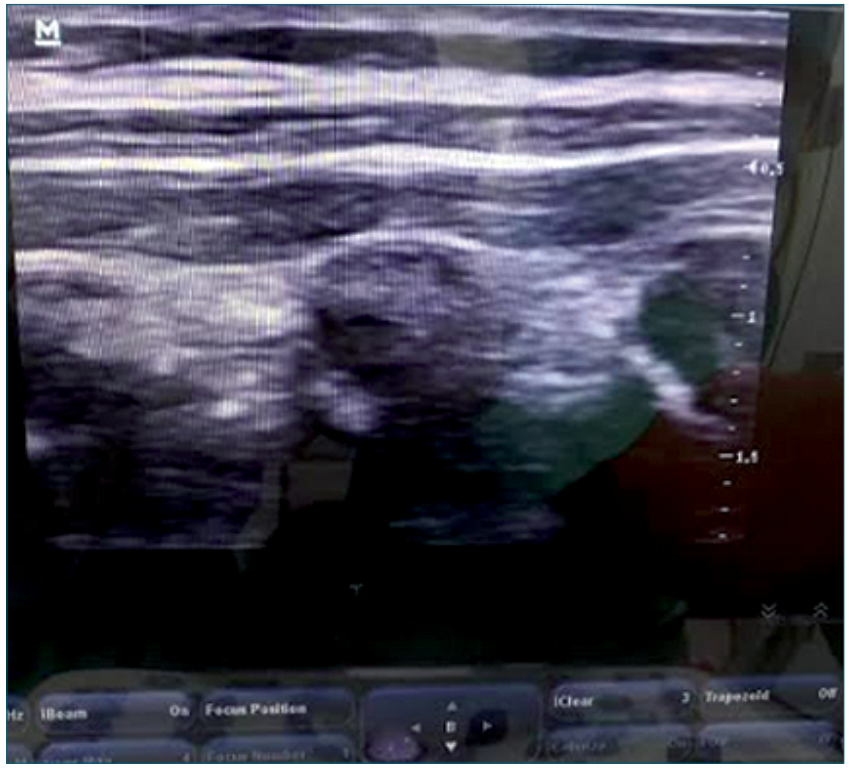

Figura 3. Planos profundos mediante ultrasonido (fuente: los autores).

sin opioides, FLACC 2/10. Estancia en UCIP de 48 h, con disminución de drenaje por sonda pleural (día 1 de 60 ml, día 2 de 24 $\mathrm{ml}$ ) y posterior retiro. Los paraclínicos de control fueron hemoglobina sin requerimientos de transfusión y gases arteriales sin trastorno ácido-base ni electrolítico. Trasladaron a la paciente a sala general y se dio de alta de manera satisfactoria al cuarto día posoperatorio.

\section{Discusión}

La aplicación del bloqueo ESP en la población adulta abarca diversos procedimientos: Manejo del dolor posoperatorio en cirugía de tórax, abdominal y de mama, así como facilidad de extubación de pacientes en UCI[2], la analgesia posoperatoria después de una cesárea[5],[6], dolor de hombro y artroplastía total de cadera[5].

Por el contrario, el reporte de uso en pacientes pediátricos es mucho más limitado; el primer caso se reportó en un paciente de 7 años durante una cirugía oncológica[3], posteriormente, un paciente de 3 años sometido a toracotomía abierta[7], un paciente de 3 años en una cirugía torácica de la pared posterior[8], cirugía de hernia inguinal en paciente prematuro de 29 semanas, quien fue operado a los dos meses de edad[9] y dos pacientes de 13 años diagnosticados con pectus excavatum y pectus carinatum respectivamente[2].

Este es el primer caso reportado en un lactante menor de 9 meses de edad, para resección de tumor en mediastino anterior de $6 \mathrm{~cm}$ de diámetro, mediante toracotomía anterolateral izquierda a través del $6^{\circ}$ espacio intercostal.

La implementación de esta técnica en pacientes pediátricos ha traído muchos beneficios, teniendo en cuenta los riesgos de neurotoxicidad relacionados con fármacos empleados en anestesia general y los efectos hasta ahora desconocidos de los fármacos neuroaxiales en el desarrollo de la médula espinal[9], adicionalmente, el uso del bloqueo ESP proporciona una alternativa libre de opioides, para controlar satisfactoriamente

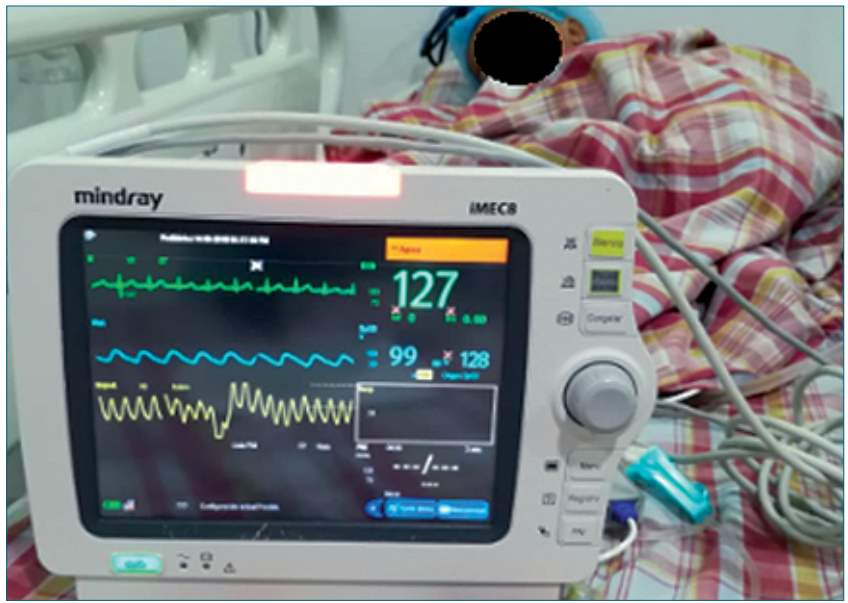

Figura 4. Ingreso a UCIP (fuente: los autores).

el dolor[7], evitando el riesgo de presentar efectos secundarios de los opioides, como náuseas y vómitos, retención urinaria y estreñimiento[2] como se evidenció en el caso de este paciente, quien fue trasladado a sala general y se dio egreso al cuarto día posoperatorio.

\section{Referencias}

1. Forero M, Adhikary SD, Lopez H, Tsui C, Chin KJ. The erector spinae plane block a novel analgesic technique in thoracic neuropathic pain. Reg Anesth Pain Med. 2016;41(5):621-7.

2. Nardiello MA, Herlitz M. Bilateral single shot erector spinae plane block for pectus excavatum and pectus carinatum surgery in 2 pediatric patients. Rev Esp Anestesiol Reanim [Internet]. Sociedad Española de Anestesiología, Reanimación y Terapéutica del Dolor; 2018;4-7. Available from: https://doi.org/10.1016/j. redar.2018.04.006

3. Muñoz F, Cubillos J, Bonilla AJ, Chin KJ. Erector spinae plane block for postoperative analgesia in pediatric oncological thoracic surgery. Can J Anesth Can d'anesthésie [Internet]. 2017;64(8):880-2. Available from: http://link.springer. com/10.1007/s12630-017-0894-0

4. Aksu C, Gürkan Y. Ultrasound guided erector spinae block for postoperative analgesia in pediatric nephrectomy surgeries. J Clin Anesth [Internet]. Elsevier Inc.; 2018;45:35-6. Available from: https://doi.org/10.1016/j.jclinane.2017.12.021

5. Selvi O, Tulgar S. Bloqueo en el plano del erector de la columna ecoguiado como causa de bloqueo motor imprevisto. Rev Esp Anestesiol Reanim [Internet]. Sociedad Española de Anestesiología, Reanimación y Terapéutica del Dolor; 2018; Available from: https://linkinghub.elsevier.com/retrieve/pii/S0034935618301105

6. Yamak Altinpulluk E, García Simón D, Fajardo-Pérez M. Erector spinae plane block for analgesia after lower segment caesarean section: Case report. Rev Esp Anestesiol Reanim [Internet]. Sociedad Española de Anestesiología, Reanimación y Terapéutica del Dolor; 2018;65(5):284-6. Available from: http://dx.doi. 
org/10.1016/j.redar.2017.11.006

7. De la Cuadra-Fontaine JC, Concha M, Vuletin F, Arancibia H. Continuous Erector Spinae Plane block for thoracic surgery in a pediatric patient. Paediatr Anaesth. 2018;28(1):74-5.

8. Hernández MA, Palazzi L, Lapalma J, Forero M, Chin KJ. Erector
Spinae Plane Block for Surgery of the Posterior Thoracic Wall in a Pediatric Patient. Reg Anesth Pain Med. 2018;43(2):217-9.

9. Hernandez MA, Palazzi L, Lapalma J, Cravero J. Erector spinae plane block for inguinal hernia repair in preterm infants. Paediatr Anaesth. 2018;28(3):298-9. 